\title{
INCLUSIÓN Y DIFERENCIACIÓN DE LA EDUCACIÓN SECUNDARIA PARA JÓVENES Y ADULTOS EN ARGENTINA. \\ UN ANÁLISIS SOBRE LA ESPECIFICIDAD DE LA OFERTA DEL PLAN FINES II
}

\author{
Julián Olivares* \\ CONICET, Universidad Nacional de General Sarmiento, Argentina \\ olivares.julian.90@gmail.com
}

Recibido: 22/06/2020 - Aceptado: 28/12/2020

\section{Resumen}

El presente artículo tiene como objetivo abordar la especificidad de la oferta del Plan de Finalización de Estudios Primarios y Secundarios para Jóvenes y Adultos II, más conocido como FinEs II, en Argentina. Para ello se avanza a través de distintos niveles de análisis, tomando en cuenta las determinaciones de la expansión de la educación secundaria en el país, para luego observar la normativa general que regula la política educativa estudiada a nivel nacional y en la provincia de Buenos Aires (donde más desarrollo tuvo el programa), las particularidades de su diseño curricular y sus diferencias con otras ofertas secundarias para jóvenes y adultos, las características de los espacios de cursada, así como también de las formas que asume el trabajo de docentes y referentes de las sedes.

Palabras clave: FinEs II - Educación secundaria - Educación de jóvenes y adultos - Inclusión Diferenciación.

\section{INCLUSION AND DIFFERENTIATION OF SECONDARY EDUCATION FOR YOUTH AND ADULTS IN ARGENTINA. AN ANALYSIS OF THE SPECIFICITY OF THE OFFER OF THE FINES II PLAN}

\begin{abstract}
The present article aims to approach the specificity of the offer of the Plan de Finalización de Estudios Primarios y Secundarios para Jóvenes y Adultos, better known as FinEs II, in Argentina. For this, it advaces through different levels of analisis, taking into account the determinations of the expansion of secondary education in the country, to later observe the general normative that regulates the educational policy studied at national level and in the province of Buenos Aires (where the program had more development), the particularities of its curricular design and their differences with other secondary offers for youth and adults, the characteristics of the study spaces, as well as the ways that the work of teachers and referents assumes.
\end{abstract}

Keywords: FinEs II - Secondary education - Education for youth and adults - Inclusion Differentiation.

\footnotetext{
* Doctorando en Historia, Universidad de Buenos Aires; Licenciado en Historia, Universidad Nacional de Luján; Profesor en Historia, I.S.P. Joaquín V. González; Becario doctoral, CONICET-UNGS.
} 


\section{Introducción}

En sintonía con una tendencia de carácter mundial, durante las últimas décadas se ha producido en Argentina una importante expansión de la secundaria, expresada entre otros aspectos en el crecimiento de la matrícula, de las tasas de escolarización y del gasto absoluto dedicado a dicho nivel (Acosta, 2012; Rivas y Dborkin, 2018). No obstante, este proceso se desarrolló mediado por una creciente diferenciación interna del sistema educativo, a partir de la descentralización educativa y la consolidación de circuitos escolares con ofertas y calidades disímiles a lo largo del territorio nacional (Braslavsky, 1989; Tiramonti, 2009). A su vez, en particular desde comienzos del siglo XXI las políticas educativas encaradas por el Estado nacional han apuntado a la inclusión de aquellos sujetos expulsados de la escuela secundaria. Dentro de la Educación de Jóvenes y Adultos (EDJA), esto llevó al surgimiento de una notable variedad de programas para la terminalidad educativa, con propuestas flexibles que buscan formar a un sector de la población trabajadora. Este fue el caso del Plan de Finalización de Estudios Primarios y Secundarios para Jóvenes y Adultos II (FinEs II).

Este trabajo tiene como objetivo explorar las características educativas del plan FinEs II. Para ello se indaga la normativa que da forma al programa en Argentina y la provincia de Buenos Aires (jurisdicción donde más desarrollo tuvo), el diseño curricular, las diferencias con otras ofertas para jóvenes y adultos como es el caso de los Centros Educativos de Nivel Secundario (CENS), las condiciones de trabajo docente y de los espacios educativos, entre otros elementos. Se busca responder a la pregunta sobre la especificidad de esta política educativa, para aportar a la comprensión sobre el papel que cumplió en la formación de trabajadores en la última década.

\section{Expansión y diferenciación interna de la educación secundaria en Argentina}

Como se dijo anteriormente, en Argentina se desarrolló durante las últimas décadas un proceso de expansión del nivel medio, que se volvió obligatorio a partir del año 2006 con la sanción de la Ley Nacional № 26.206, más conocida como Ley de Educación Nacional (LEN). Dicho movimiento implicó entre otros aspectos un aumento de la matrícula y de las tasas de escolarización, así como del gasto absoluto dedicado al sostenimiento de la secundaria. Este crecimiento fue de la mano de un incremento de aquellos contenidos de corte general, por encima de la formación especializada (Río y Schoo, 2016).

Pero a la par, el sistema educativo avanzó desde fines de la década del '60 hacia una creciente segmentación y fragmentación, que derivaron entre otros aspectos en la profundización de circuitos educativos cada vez más diferenciados ${ }^{1}$. De este modo, la descentralización educativa, la desregulación del sector privado y notables niveles de abandono, repitencia y sobreedad escolar han sido características centrales de la escuela secundaria argentina (Acosta, 2012; Tiramonti, 2019). Uno de los resultados ha sido la consolidación de un caudal significativo de jóvenes y adultos que no han llegado a completar el secundario, aun cuando este ha sido sancionado como obligatorio (Riquelme et. al. 2018). Como plantean distintos autores, esta progresiva diferenciación interna del sistema educativo debe analizarse como expresión de las transformaciones en las condiciones de reproducción de los trabajadores durante las últimas décadas. De esa manera, es el proceso de acumulación de capital en Argentina el que ha determinado una demanda por acentuar cada vez más las diferencias entre los atributos productivos de las distintas porciones de la fuerza laboral, con su consiguiente efecto en el acceso a la educación (Hirsch, 2020; Iñigo y Río, 2017; Mendonça y Pérez Trento, 2020)².

Como expresión particular de este proceso, la educación secundaria de jóvenes y adultos en el país ha mostrado un notable crecimiento y dinamismo durante las últimas décadas. Esta tendencia no solo se reflejó en un aumento del número de alumnos que asisten a la modalidad en el nivel medio, sino en importantes cambios en lo que respecta al tipo de oferta, como así también en la composición de su matrícula (Brusilovsky y Cabrera, 2012; De la Fare, 2013; Finnegan, 2016). No obstante, si bien la EDJA estuvo en sus orígenes fuertemente centralizada por el Estado nacional (Rodríguez, 2009), al igual que sucedió con el resto del sistema educativo, la misma avanzó en las últimas décadas hacia mayores niveles de diferenciación y a caracterizarse por la dispersión curricular, coexistiendo de manera creciente diversas ofertas educativas para jóvenes y adultos que tienen distintos diseños curriculares, aun cuando sus objetivos, destinatarios e incluso espacios geográficos sean los mismos (Brusilovsky y Cabrera, 2006; Montesinos et. al. 2010).

La educación de jóvenes y adultos en Argentina pasó así a desempeñar sobre todo una función compensatoria, focalizada y degradada que apuntó a permitir la alfabetización o la terminalidad de la primaria o secundaria de aquellos sujetos que quedaron por fuera del sistema educativo. Compensatoria

\footnotetext{
'La segmentación está determinada por la existencia de diferentes redes o circuitos de escolarización dentro del sistema educativo. Si bien los sistemas educativos se encuentran segmentados desde sus orígenes, las transformaciones educativas en las décadas recientes han dado lugar a una creciente diferenciación entre dichos circuitos donde se realiza el proceso de formación escolar de la población. Por otra parte, la fragmentación remite a una ruptura de la unidad del sistema educativo como producto de la descentralización educativa, en donde cada institución pasa a estar separada del conjunto y teniendo como referencia al contexto territorial inmediato y la población destinataria. Un análisis de estos conceptos puede verse en Botinelli (2017).

2 Se entiende por atributos productivos universales a aquellas aptitudes, capacidades y saberes que todos los sujetos deben poseer para participar del proceso general de reproducción social, tales como la lectocomprensión, el pensamiento abstracto, la disciplina, la capacidad de trabajar colectivamente, etc. Atributos generales que exceden aquellas facultades más inmediatas que cada trabajo particular requiere (Hirsch e Iñigo, 2005)
} 
porque cumplió el papel de favorecer el reingreso a la escuela a quienes la abandonaron con anterioridad. Focalizada porque esta meta se enfocó en general a modo de asistir puntualmente a la población empobrecida, para la cual se requieren mecanismos educativos específicos en pos de garantizar su escolarización efectiva (menor carga horaria o contenidos, flexibilización del régimen académico, etc.). El resultado fue un proceso educativo de menor calidad que el de la educación común, y por lo tanto degradado.

\section{Evolución de la normativa de FinEs II}

La Ley de Educación Nacional del año 2006 decretó la obligatoriedad de la escolaridad secundaria y la creación de una modalidad específica de educación permanente de jóvenes y adultos, así como la necesidad de constitución de planes de terminalidad educativa. Al igual que sucedió con el resto de la educación, la LEN destacó como relevantes para la EDJA la formación ciudadana y la educación para el mundo del trabajo, las cuales a la vez fueron abordadas desde una concepción flexible de la enseñanza, teniendo en cuenta los individuos hacia los cuales está orientada. Estas cuestiones fueron ratificadas en la provincia de Buenos Aires a partir de la sanción de la Ley de Educación Provincial en el año 2007.

En sintonía con estas definiciones, la Resolución 22/07 del Consejo Federal de Educación (CFE) aprobó el documento titulado "Lineamientos para un Plan Federal de Educación Permanente de Jóvenes y Adultos 2007/2011", cuya aplicación se llevó adelante junto a otros ministerios como el de Desarrollo Social, como también entidades barriales, sindicatos, etc. En el anexo de dicho documento se afirmó la relevancia de identificar las particularidades de los adultos que no hayan completado los requisitos educativos fijados por la LEN y de la implementación de los dispositivos necesarios para avanzar en ellos.

Un año más tarde la Resolución 917/08 del Ministerio de Educación de la Nación sancionó la normativa general del "Plan de Finalización de Estudios Primarios y Secundarios - FinEs", para ser encauzada desde la Secretaría de Educación. Esta resolución se encargó de asentar las bases para la fase inicial del Plan FinEs, que comenzó en el 2008 y estuvo destinada solo a adultos mayores de 18 años que hubiesen terminado la cursada del último año del nivel medio y adeudasen materias. Al mismo tiempo se delimitó un segundo momento, entre el año 2009 y 2011, en el cual se ampliaría a todos los adultos que no hubieran empezado o acabado la primaria o secundaria, convirtiéndose en una oferta nueva de cursada para esos niveles.

Posteriormente, la Resolución 66/08 del Consejo Federal de Educación aprobó dichos criterios y la aplicación de la primera etapa del programa. En la provincia de Buenos Aires, esto se vio reflejado en el Convenio Marco 299/08 del Ministerio de Educación de la Nación, que estableció el comienzo de FinEs en la jurisdicción, así como la Resolución 4122/08 de la Dirección General de Cultura y Educación (DGCyE) bonaerense, centrada en su desarrollo en articulación con una oferta similar preexistente denominada Centros de Orientación y Apoyo (COA). También se planteó allí el despliegue de la segunda fase del mismo.

A escala nacional, en el año 2009 se dio otro avance con la Resolución 87/09 del CFE, la cual aprobó para su discusión los documentos "Educación Permanente de Jóvenes y Adultos - Documento Base" y "Lineamientos curriculares para la Educación Permanente de Jóvenes y Adultos". El primero apuntaba a asentar los cimientos de la EDJA, desarrollando un recorrido histórico de lo que fue la modalidad de jóvenes y adultos, haciendo hincapié en la particularidad económica de los destinatarios (sujetos atravesados por problemáticas tales como la pobreza y la marginación, que buscan la titulación por diversas motivaciones) y en el imperativo de crear trayectos diferenciados para los mismos. De allí que se planteó la importancia de crear formatos flexibles que posibiliten su acceso y permanencia en la escuela. El segundo documento avanzó en una propuesta puntual. Entre los aspectos centrales se clarificó el diseño curricular modular, en consonancia con la idea de autonomía, accesibilidad y acreditación parcial del recorrido académico. La carga horaria se fijó como carga de trabajo total, contemplando la posibilidad de distintos esquemas (tanto presenciales como semipresenciales o a distancia) y contando el trabajo fuera del aula. La delimitación de estas áreas quedó en manos de las jurisdicciones.

Estos documentos se ratificaron con la Resolución 118/10 del CFE, terminando de construir el cuerpo normativo que dio origen al programa FinEs II como trayecto educativo (a diferencia de la anterior oferta de FinEs destinada a deudores de materias). Esta resolución agregó, a su vez, que dichos fundamentos fueran la base sobre la cual cada una de las jurisdicciones del país creara sus planes de estudio en un plazo máximo de 3 años.

En la provincia de Buenos Aires, la Resolución 3520/10 de la DGCyE fue la responsable de establecer el diseño curricular provincial de esta segunda fase para la escolaridad media. La misma tomó como referencia principal a la Resolución $6321 / 95$ de la DGCyE, que regula los CENS. Explicitó además una vigencia de cinco años para la aplicación de FinEs II, que su funcionamiento dependería de la Subsecretaría de Educación y que los destinatarios serían mayores de 18 años pertenecientes a cooperativas del plan Argentina Trabaja o entidades gremiales.

En el anexo se definió como ofertas a las orientaciones de Ciencias Sociales y Gestión y Administración. Se delimitó un plan de estudios de tres años, con dos cuatrimestres cada uno. Se dio la opción de cursar dos o cuatro días por semana (pudiendo entonces optar por hacer un máximo de dos cuatrimestres en simultáneo). Esto implicó que se podría hacer el trayecto entero del secundario en 1 año y medio, sin correlatividades entre las materias. Los estudiantes debían cumplir con el $75 \%$ de asistencia (es decir, 4 faltas como el máximo permitido) para sostener su condición de regular, aunque en los hechos esto fue más flexible y se adaptó a la particularidad de la población destinataria. La cursada se estructuró en 5 materias cuatrimestrales (30 materias en total), con una carga horaria de 13 hs cátedra semanales. La 
Disposición 27/11 de la DGCyE aclaró los contenidos de las materias de ambas especialidades. Los títulos quedaron a cargo de los CENS de cada partido, lo cual como se verá más adelante implicó un ahorro significativo para el Estado al no crear un nuevo aparato administrativo para FinEs II.

Con posterioridad, en el 2012 la Resolución 444/12 de la DGCyE aprobó en la provincia de Buenos Aires el "Plan Provincial de Finalización de Estudios Obligatorios", dando continuidad a FinEs II. En la misma se resaltó que las sedes podrían estar tanto en escuelas como en entidades del Estado, organizaciones gremiales, sociales, empresariales, universidades y ONG, entre otras. A su vez, la población destinataria se extendió a todos los mayores de 18 años que no hubieran completado el secundario. Ese año la Resolución Ministerial 1042/12 prolongó el desarrollo de FinEs II de 2012 a 2015, y más adelante en el 2016 la Resolución 178/16 del Ministerio de Educación de la Nación sancionó la continuidad del programa durante el período 2016-2019.

Un cambio significativo se produjo en el año 2017 en la provincia de Buenos Aires con la Resolución 713/17 de la DGCyE, que modificó aspectos fundamentales de lo establecido en la Resolución 3520/10, al mismo tiempo que suprimió la Resolución 444/12. Además de ratificar la persistencia de FinEs II hasta el 2019, anuló la opción de cursar dos cuatrimestres de manera simultánea y decretó un incremento en la carga horaria, la cual aumentó de 13 a 18 hs cátedra semanales. Por esa razón, el número de días pasó a ser tres por semana. Se fijó el pago del salario docente por las horas desempeñadas frente a curso y se centralizó el plan en el inspector de adultos y los directores de los CENS. A la vez, en el 2018 los CENS en la jurisdicción comenzaron a organizarse en base al esquema "18+8", pasando a tener 18 hs de cursada presenciales y 8 hs de tutorías (Resolución 2280/17 de la DGCyE). De modo que se vio una suerte de equiparación, al menos en el aspecto horario.

Finalmente, la resolución 146/2020 del Ministerio de Educación de la Nación aprobó la implementación del Plan FinEs 2020, que dio continuidad a esta política educativa, a la vez que introdujo entre otros cambios, la posibilidad de aplicar cursadas de forma virtual y de que las sedes del plan funcionen en otros establecimientos de educación de jóvenes y adultos.

\section{Comparación de los diseños curriculares de FinEs II y CENS}

La diferenciación educativa en el nivel secundario se manifiesta con claridad en el diseño curricular del plan FinEs II en la provincia de Buenos Aires. Si bien el mismo se sustenta en la resolución del año 1995 que regula los CENS, tiene una marcada reducción horaria en relación a esa oferta de educación media común y de adultos.

El diseño curricular de los CENS, nacido de la Resolución 6321/95 de la DGCyE, fija para la orientación del Bachillerato en Ciencias Sociales (la que mayormente fue aplicada y será analizada a continuación) un plan de estudios con una asignación total de 26 hs cátedra semanales, el cual se organiza en 3 años (con 2 cuatrimestres cada uno). El 1er año dedica 18 de esas horas a las materias de formación general $(69 \% \text { del total) })^{3}, 6$ horas a las materias de formación orientada $(23 \% \text { del total) })^{4}$ y 2 horas a las especializadas $(8 \% \text { del total })^{5}$. El segundo año dedica 15 horas a la formación general $(58 \% \text { del total })^{6}, 6$ horas a las materias de formación orientada $(23 \% \text { del total })^{7}$ y 5 horas a las especializadas (19\% del total). El tercer año dedica de nuevo 15 horas a la formación general $(58 \% \text { del total })^{8}, 6$ horas a las materias de formación orientada ( $23 \%$ del total) ${ }^{9}$ y 5 horas a las especializadas ( $19 \%$ del total).

Como se explicó antes, el plan de estudios original de FinEs II en la provincia de Buenos Aires (Resolución 3520/10 de la DGCyE), tomó de modo directo la resolución de 1995 de los CENS. Por ende, se respetaron las materias, el porcentaje y la carga horaria semanal específica de las mismas ${ }^{10}$. Sin embargo, la normativa determinó para la cursada una asignación de 13 hs totales por semana. Las asignaturas dejaron de ser anuales y pasaron a ser cuatrimestrales, en tanto se redujo en un $50 \%$ su carga horaria con respecto a los $\mathrm{CENS}^{12}$. Es decir que el contenido anual de cada materia se vio reducido por la

\footnotetext{
${ }^{3}$ Las materias son Biología (3 hs), Educación Cívica (2 hs), Historia y Geografía (3 hs), Informática (2 hs), Inglés (2 hs), Lengua y Literatura (3 hs), Matemática (3 hs).

${ }^{4}$ Las materias son Psicología ( $3 \mathrm{hs}$ ) y Sociología ( $3 \mathrm{hs}$ ).

${ }^{5}$ Materias a ser definidas por los establecimientos educativos según su proyecto institucional.

${ }^{6}$ Las materias son Física (2 hs), Historia y Geografía (3 hs), Informática (2 hs), Inglés (2 hs), Lengua y Literatura (3 hs) y Matemática (3 hs).

${ }^{7}$ Las materias son Ciencias Políticas ( $3 \mathrm{hs}$ ) y Metodología de la Investigación (3 hs).

${ }^{8}$ Las materias son Informática (2 hs), Inglés ( 2 hs), Lengua y Literatura (3 hs), Matemática ( 3 hs), Problemática Social Contemporánea (3 hs) y Química (2 hs).

${ }^{9}$ Las materias son Comunicación y Medios (3 hs) y Filosofía (3 hs).

${ }^{10}$ Para la orientación de Ciencias Sociales se establecieron como materias de la especialización: en 1er año Economía Social (2 hs); en 2do año Estado y Políticas Públicas ( $3 \mathrm{hs})$, junto con Políticas Públicas y Derechos Humanos (2 hs); en 3er año Diseño y Desarrollo de Proyectos (2 hs), junto con Estado y Nuevos Movimientos Sociales (3 hs).

${ }^{11}$ En el 1er cuatrimestre de 1er año se dictan las materias Biología, Historia y Geografía, Informática, Inglés y Lengua y Literatura. En el 2do cuatrimestre de 1er año se dictan Economía Social, Educación Cívica, Matemática, Psicología y Sociología. En el 1er cuatrimestre del 2do año se dictan Ciencias Políticas, Física, Historia y Geografía, Inglés y Lengua y Literatura. En el 2do cuatrimestre del 2do año se dictan Estado y Políticas Públicas, Informática, Matemática, Metodología de la Investigación y Políticas Públicas y Derechos Humanos. En el 1er cuatrimestre del 3er año se dictan Diseño y Desarrollo de Proyectos, Informática, Lengua y Literatura, Matemática y Problemática Social Contemporánea. En el 2 do cuatrimestre del 3er año se dictan Comunicación y Medios, Estado y Nuevos Movimientos Sociales, Filosofía, Inglés y Química.
} 
mitad. Así, en lugar de tener Historia y Geografía, Lengua y Literatura, Matemática, etc. durante todo el año, se pasó a tenerlas sólo un cuatrimestre, con un total de 16 clases, junto a otros 2 días de compensación de notas en el Curso de Recuperación y Evaluación Continua (CREC).

Cuadro 1. Comparación de la oferta educativa entre CENS y FinEs II. Provincia de Buenos Aires.

\begin{tabular}{|c|c|c|c|c|}
\hline & $\begin{array}{c}\text { CENS (1995-2017) } \\
\text { Res. 6321/95 }\end{array}$ & $\begin{array}{c}\text { FinEs II (2010-2016) } \\
\text { Res. 3520/10 }\end{array}$ & $\begin{array}{c}\text { FinEs II (2017- } \\
\text { presente) } \\
\text { Res. 317/17 }\end{array}$ & $\begin{array}{c}\text { CENS (2018- } \\
\text { presente) } \\
\text { Res. 2280/17 }\end{array}$ \\
\hline $\begin{array}{c}\text { Cantidad de } \\
\text { años }\end{array}$ & 3 & 3 & 3 & 3 \\
\hline $\begin{array}{c}\text { Cantidad de } \\
\text { horas } \\
\text { semanales }\end{array}$ & $\begin{array}{c}26 \text { hs cátedra } \\
\text { presenciales }\end{array}$ & $\begin{array}{c}13 \text { hs cátedra } \\
\text { presenciales }\end{array}$ & $\begin{array}{c}18 \text { hs cátedra } \\
\text { presenciales }\end{array}$ & $\begin{array}{c}18 \text { hs cátedra } \\
\text { presenciales y } 8 \\
\text { hs cátedra de } \\
\text { tutorías }\end{array}$ \\
\hline $\begin{array}{c}\text { Cantidad de } \\
\text { días }\end{array}$ & 5 & 2 & 3 & 5 \\
\hline $\begin{array}{c}\text { Cantidad de } \\
\text { asignaturas }\end{array}$ & 30 & 30 & 30 & 30 \\
\hline Modalidad & Anual & Cuatrimestral & Cuatrimestral & Anual \\
\hline
\end{tabular}

Fuente: elaboración propia en base a Resoluciones 6321/95, 3520/10, 713/17 y 2280/17 de la DGCyE.

Esta situación es señalada por otros autores como una de las mayores problemáticas que presenta el programa (Kurlat y Lozano, 2014; López, 2015; Viego, 2015). Es que una reducción de los tiempos de estudio de tal magnitud atenta contra la posibilidad de alcanzar los objetivos mínimos establecidos por las propias resoluciones ministeriales. Ante esto los profesores deben optar por realizar planificaciones y proyectos que se adapten, ya sea recortando contenidos o abordándolos de un modo más superficial ${ }^{12}$. Se ve entonces una diferenciación de la educación que adquieren los alumnos en relación a los CENS.

Como se dijo antes, este diseño curricular en la provincia de Buenos Aires se modificó con la Resolución 713/17 de la DGCyE, la cual se implementó a partir del año 2017. La misma fijó un incremento a 18 hs semanales, sumando un día de cursada. Es decir, un aumento del $38 \%$ de la carga horaria total. En contraposición a la primera oferta de FinEs II, que respetaba los porcentajes de los CENS, se dio el crecimiento en las materias de formación general, en desmedro del resto.

En consecuencia, el 1er año dedica ahora un $75 \%$ de la asignación horaria a las materias de formación general, un $19 \%$ a las de formación orientada y un $6 \%$ a las especializadas. El segundo año dispone un 64\% a las materias de formación general, un $19 \%$ a las de formación orientada y un $17 \%$ a las especializadas. Finalmente, el tercer año asigna un $64 \%$ a las de formación general, un $17 \%$ a las de formación orientada y un $19 \%$ a las especializadas.

\footnotetext{
${ }^{12} \mathrm{El}$ desarrollo de los proyectos docentes, como mediación entre el diseño curricular y la enseñanza concreta, queda fuera de este escrito. Se considera de todas maneras que observar la carga horaria otorga suficiente claridad en este aspecto. Sin embargo, es un camino abierto a futuras investigaciones.
} 
Gráfico 1. Comparación de porcentajes de formación general, orientada y especializada en CENS, FinEs II (2010-2016) y FinEs II (2017-presente) con orientación en Ciencias Sociales según año de cursada. Provincia de Buenos Aires

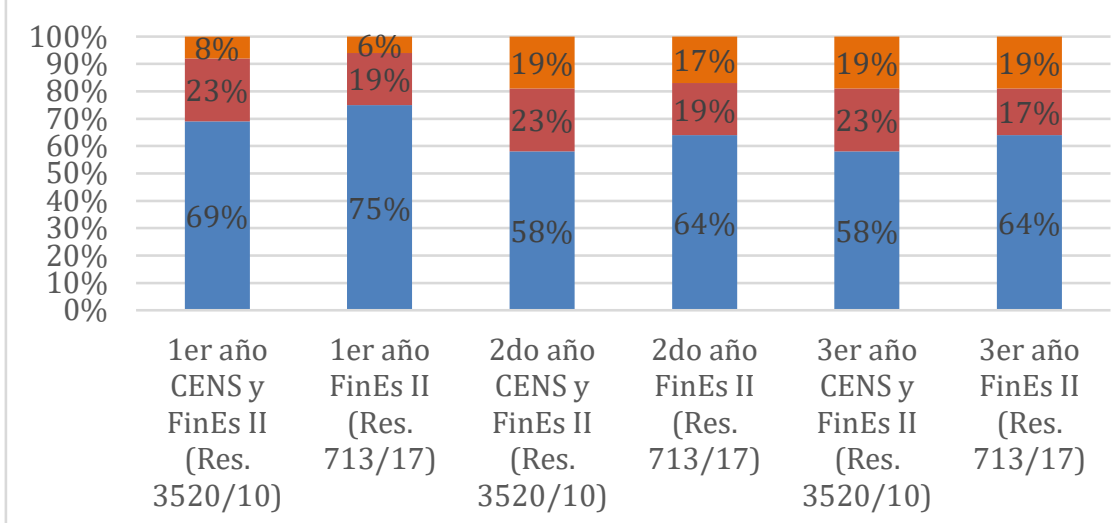

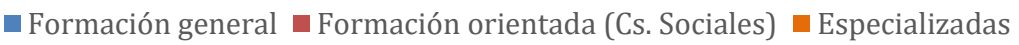

Fuente: elaboración propia en base a Resoluciones 6321/95, 3520/10 y 713/17 de la DGCyE.

Este cambio no afectó de manera homogénea a las materias. Mientras que algunas de ellas no incrementaron su carga horaria en absoluto ${ }^{13}$, otras lo hicieron en apenas 1 hora cátedra ${ }^{14}$. y otras la duplicaron ${ }^{15}$. Las materias en las cuales se enfocó el aumento fueron Lengua y Literatura junto con Matemática, las cuales concentraron un $60 \%$ de esas $5 \mathrm{hs}$ del incremento de la carga horaria total que tuvo el diseño curricular (por el incremento de 13 a 18 hs semanales), al mismo tiempo que individualmente tuvieron un acrecentamiento del 100\% (pasando ambas de 3 a 6 hs).

Gráfico 2. Comparación de porcentajes de la carga horaria del 1ํaño de FinEs II (diseños curriculares de 2010 y 2017) con orientación en Ciencias Sociales según materias. Provincia de Buenos Aires

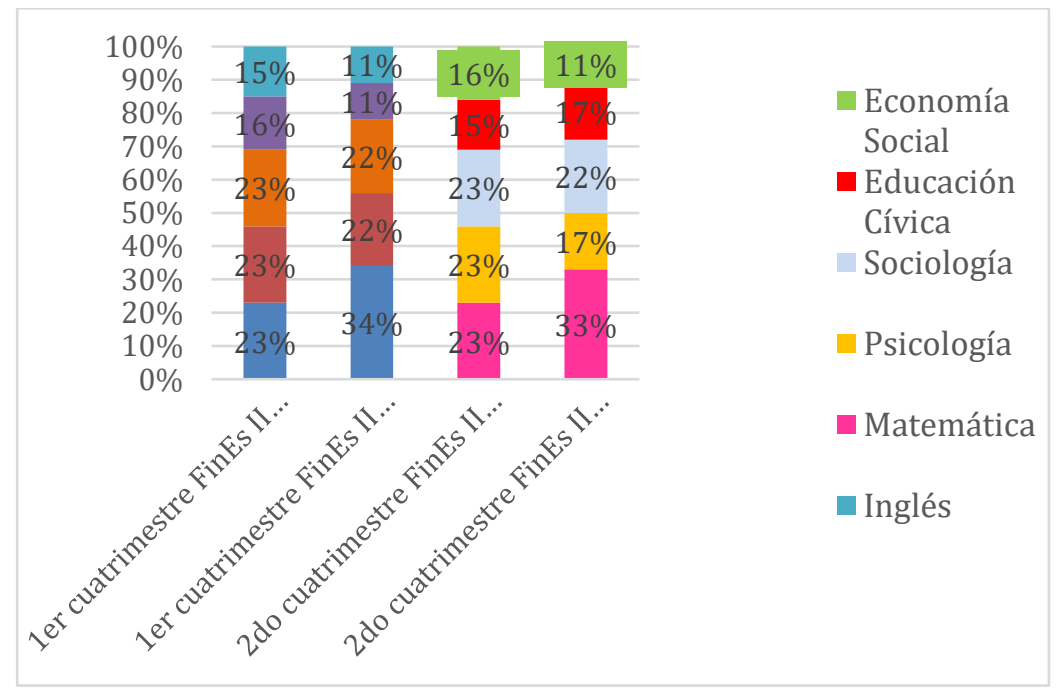

Fuente: elaboración propia en base a Resoluciones 3520/10 y 713/17 de la DGCyE.

\footnotetext{
${ }^{13}$ Es el caso de Ciencias Políticas, Comunicación y Medios, Economía Social, Filosofía, Informática e Inglés en los 3 años, Políticas Públicas y Derechos Humanos, y Psicología.

${ }_{14}$ Es el caso de Biología, Comunicación y Medios, Diseño y Desarrollo de Proyectos, Educación Cívica, Estado y Nuevos Movimientos Sociales, Estado y Políticas Públicas, Física, Historia y Geografía en 1er y 2do año, Metodología de la Investigación, Problemática Social Contemporánea, Sociología y Química.

${ }^{15}$ Es el caso de Lengua y Literatura junto con Matemática en los 3 años. Como ajuste, Matemática en 3er año pasa al 2do cuatrimestre, rotando con Química (que pasa al 1ero).
} 
REVISTA DE LA ESCUELA DE CIENCIAS DE LA EDUCACIÓN, AÑO 17, NRO. 16, VOL. 2, JULIO A DICIEMBRE DE 2021. PÁGINAS 26-36. ISSN 2362-3349 (EN LÍNEA). INCLUSIÓN Y DIFERENCIACIÓN DE LA EDUCACIÓN SECUNDARIA PARA JÓVENES Y ADULTOS EN ARGENTINA. UN ANÁLISIS SOBRE LA ESPECIFICIDAD DE LA OFERTA DEL PLAN FINES II. JULIÁN OLIVARES.

Gráfico 3. Comparación de porcentajes de la carga horaria del 2º año de FinEs II (diseños curriculares de 2010 y 2017) con orientación en Ciencias Sociales según materias. Provincia de Buenos Aires

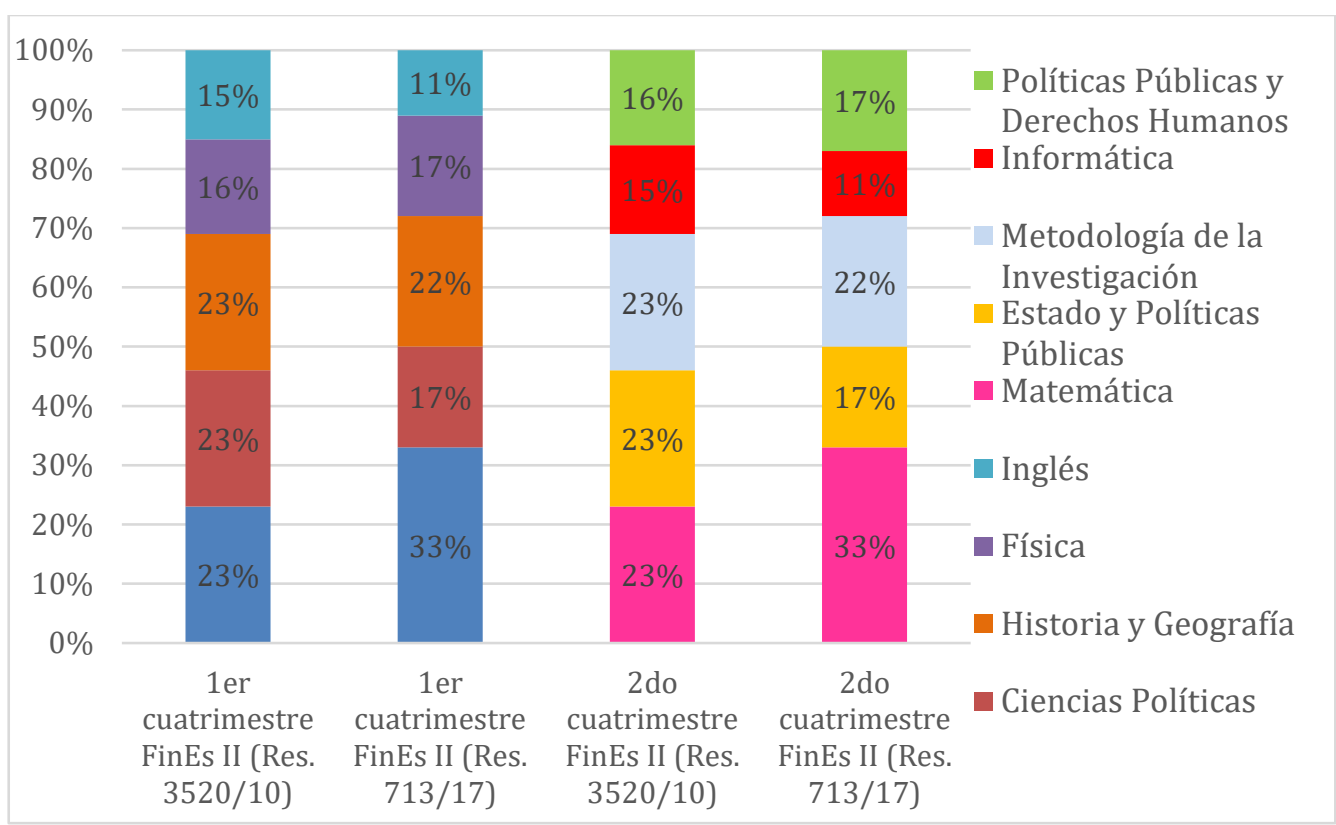

Fuente: elaboración propia en base a Resoluciones 3520/10 y 713/17 de la DGCyE.

Gráfico 4. Comparación de porcentajes de la carga horaria del 3o año de FinEs II (diseños curriculares de 2010 y 2017) con orientación en Ciencias Sociales según materias. Provincia de Buenos Aires

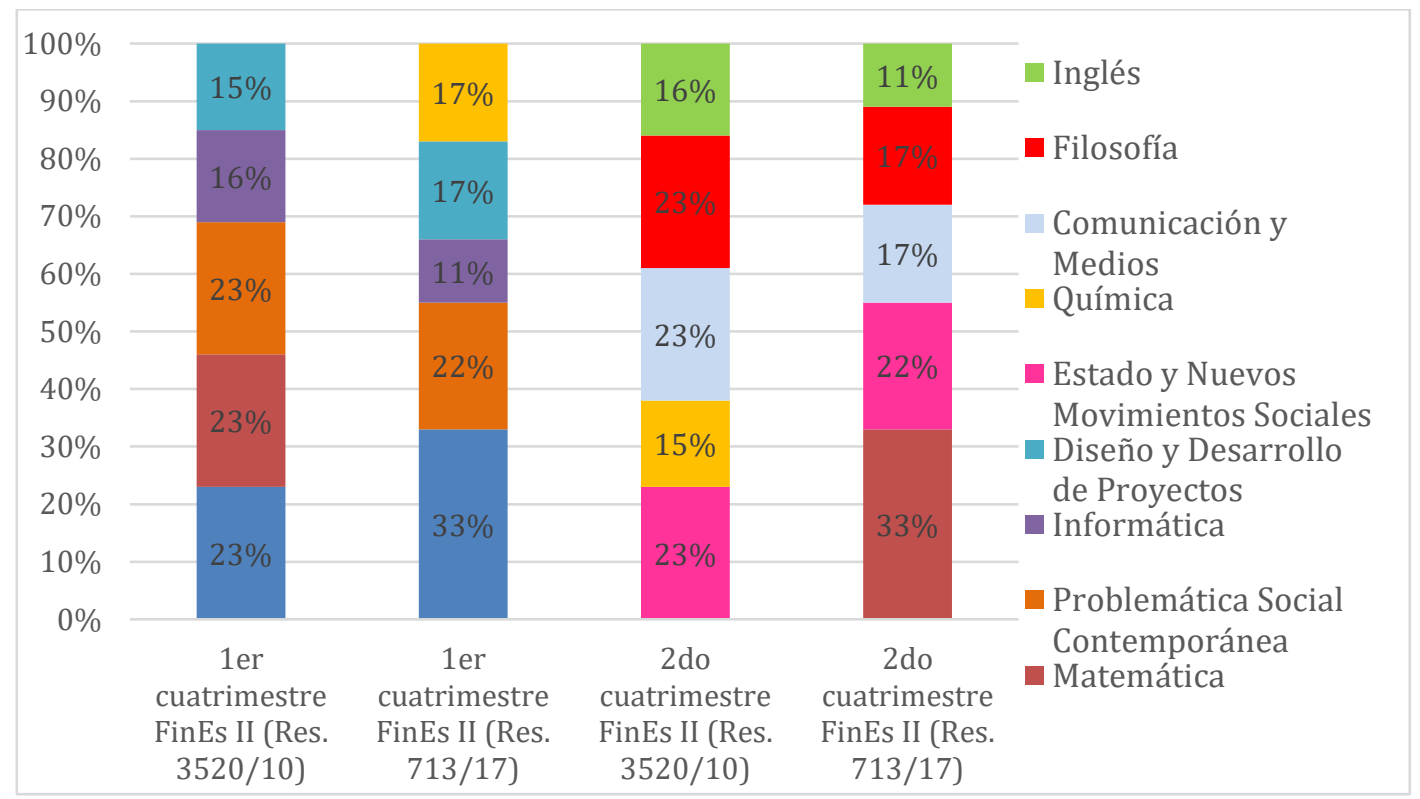

Fuente: elaboración propia en base a Resoluciones 3520/10 y 713/17 de la DGCyE.

No obstante, al parecer la flexibilización de la oferta educativa no ha evitado el pronunciado abandono escolar, elemento que ha acompañado la expansión del secundario en Argentina. Por el contrario, si bien ha permitido la inclusión de miles de jóvenes y adultos dentro del sistema educativo, por lo visto el plan FinEs II tiene una enorme deserción escolar, que puede llegar a ser incluso más elevada que la que ocurre en el resto del sistema educativo (Olivares, 2018). 


\section{Espacios en los cuales se desarrolla el proceso educativo}

Como se señala en la normativa, FinEs II se desarrolla tanto en escuelas como en organizaciones sindicales, barriales, iglesias, casas de familia, centros culturales o clubes de barrio, muchas veces alejados del centro de cada municipio. Esto acompaña el planteo de una oferta inclusiva y flexible, que garantice el acceso a la población hacia la cual está destinada. Se trata de espacios cuya ubicación sea práctica para sus horarios laborales o que sean cercanos a las viviendas de los alumnos, por ejemplo. Esto se ve reflejado en la valoración positiva que hacen los mismos estudiantes, puesto que el programa facilita su reingreso y finalización de los estudios secundarios. Al respecto, hay autores que han resaltado que este desarrollo del plan FinEs a partir del vínculo entre el Estado y las organizaciones sociales, políticas o sindicales retoma elementos clásicos de la educación de adultos en Argentina (Levy y Rodríguez, 2015).

Para algunos autores esto explica también el gran despliegue que tuvo el programa FinEs II en la provincia de Buenos Aires, jurisdicción en la cual las ofertas tradicionales de la EDJA (los CENS por ejemplo) suelen ubicarse en sedes céntricas, siendo muy reducidos los anexos en barrios periféricos (Finnegan y Montesinos, 2016). Según el Informe N. 96 de la Jefatura de Gabinete de Ministro al Senado del 2016 ese año la matrícula de FinEs II en todo el país era de 164.639 estudiantes, de los cuales 83.928 (el $51 \%$ ) se ubicaban en dicha provincia.

Sin embargo, las circunstancias que facilitan el acceso a la escuela secundaria, deterioran las condiciones de cursada. Es fundamental tener en cuenta que el Estado prácticamente no destina recursos para las sedes, las cuales deben autosustentarse con el esfuerzo de los referentes y la comunidad educativa. Esta situación aplica hasta para lo más básico como pagar los servicios de la sede o contar con artículos de limpieza. Desde bonos, donaciones, rifas, actividades para recolectar dinero, una cooperadora o aportes directos son cuestiones regulares en cada uno de los espacios (Burgos, 2015; De Luca, 2017; Esper, 2017).

La bibliografía específica sobre el plan FinEs II muestra que, con excepción de aquellas que funcionan en escuelas, muchas de las sedes se establecen en espacios que no poseen todos los componentes requeridos para llevar adelante el proceso educativo. Desde falta de insumos básicos como pizarrones o mobiliario adecuado, estufas o ventiladores, aulas que no están en condiciones, o la ausencia de equipamiento tecnológico necesario para aplicar el diseño curricular. Un claro ejemplo es la carencia de computadoras, ante lo cual la materia Informática termina siendo reducida a algo meramente teórico salvo cuando se aprende con algunos equipos portátiles del docente o de algún alumno (Kurlat y Lozano, 2014). Cabe aclarar que FinEs II no ha entrado dentro de los beneficios del programa Conectar Igualdad y la distribución de netbooks. Al unísono, esto realiza una diferenciación al interior del plan, entre las sedes de barrio, por lo común más precarizadas, y las sedes céntricas, que se ubican en establecimientos educativos.

\section{Condiciones de trabajo de docentes y referentes}

La especificidad del programa no está dada sólo por la flexibilidad del diseño curricular y los espacios en los que se cursa en relación a otras ofertas existentes para jóvenes y adultos. Las condiciones de trabajo de los docentes también son señaladas por su elevado grado de precarización por diversos autores (Burgos, 2015; Facioni et al., 2013; Giménez e Inda, 2015; Huenchunao y Muñoz, 2015). Al respecto, algunos investigadores han abordado la percepción subjetiva de los profesores sobre esta misma situación (González Porcella y Pereyra, 2015; Kurlat y Lozano, 2014), así como sus posibilidades de acción y organización sindical (Komañski, 2016).

Tal como lo fija la normativa, los profesores de FinEs II son considerados tutores, siendo su salario pagado por el gobierno nacional. Su contratación se da de manera cuatrimestral, en situación de revista provisional, eliminando la estabilidad docente. Este hecho provoca que cada cuatrimestre, antes del comienzo de las clases, todos deban concurrir a los actos públicos para cubrir la totalidad de las materias, no sabiendo si van a llegar a tomar lo que tenían con anterioridad ${ }^{16}$. A la par determina un notorio grado de inestabilidad, así como un impedimento para cobrar a término, ya que cada ciclo se debe reingresar al sistema ${ }^{17}$. A su vez, no se conserva el puesto durante los recesos de verano e invierno. Por ello las vacaciones no se pagan como el resto de la docencia en condición provisional y tampoco se conserva la obra social. Asimismo, el salario no contempla ruralidad ni tampoco acumula antigüedad para otras ramas, como las escuelas secundarias comunes o técnicas (Esper, 2017; Viego, 2015).

Otro aspecto destacado es la flexibilización de tareas que corresponden al cuerpo docente. En FinEs II los educadores deben pasar lista, elevar las notas a las autoridades de modo presencial, etc. Actividades que en las ofertas tradicionales de la escuela media atañen por ejemplo a lo que sería un preceptor (Kurlat y Lozano, 2014). Esta flexibilización laboral se aplica además en cuanto a las inasistencias, puesto que no existe régimen de licencias extraordinarias, teniendo cada docente que recuperar las clases perdidas, por lo usual en otro día u horario (Facioni et. al. 2013). El programa no dispone tampoco de un gabinete psicopedagógico que pueda dar respuesta a las diferentes problemáticas que surgen en lo cotidiano. Nuevamente aquí el Estado realiza un importante ahorro de recursos, al mismo tiempo que recarga con estas tareas a los docentes.

\footnotetext{
${ }^{16}$ Cada cargo docente se consigue en los actos públicos, que son instancias administrativas a cargo de la Secretaría de Asuntos Docentes de cada municipio, donde se cubren los cursos según un listado ordenado por el puntaje de cada profesor (que se obtiene en base al título, antigüedad en la docencia, etc.).

${ }^{17}$ En el sistema docente existe una diferencia de meses entre el momento donde se toman las horas en acto público y aquel en el que se tiene el alta para comenzar a cobrar el salario correspondiente a dicho cargo.
} 
Esto conforma en general un perfil del plantel constituido por trabajadores que en su gran mayoría recién ingresan al oficio, con menor puntaje, y que ven a FinEs II como una posibilidad laboral, frente a las dificultades para insertarse en la escuela secundaria común o los CENS, donde la competencia es más fuerte. Esto también tiene un impacto cualitativo en la enseñanza, en tanto por lo general los profesores menos experimentados no son los que tienen las mejores herramientas para otorgar una educación de mayor calidad.

En relación a la contratación, el desarrollo del programa fue evolucionando desde listados armados por la coordinación del plan, en los cuales podían estar profesores recibidos o cursando, así como maestros y profesionales idóneos sin título vinculante (contando en el puntaje tanto el título docente como el proyecto pedagógico y el Currículum Vitae), hasta un listado oficial centralizado por la Secretaría de Asuntos Docentes con puntaje en base al título.

Un aspecto relevante a tener en cuenta, poco tomado por la bibliografía, es que la precarización no se extiende sólo a los docentes, sino que el programa se sostiene en gran medida por el trabajo no remunerado de los referentes de cada espacio educativo. Tanto el mantenimiento de las sedes como de la matrícula es garantizado por ellos, los cuales no cobran un salario por su desempeño. Sus tareas se corresponden con las de un preceptor, así como también del personal jerárquico de una escuela, ya que se encargan de todo el aspecto administrativo (recibir documentación, gestionar la sede, tener un vínculo fluido con las autoridades, resolver problemáticas de los alumnos, etc.).

Esta modalidad de trabajo precario se sustenta con un discurso desde la vocación y la militancia, la cual demanda un compromiso político con el programa (Finnegan y Brunetto, 2014), pero que a la vez representa con total claridad un empleo permanente realizado de manera gratuita. Junto con la precarización de los espacios de estudio, este abaratamiento de la fuerza laboral es uno de los pilares sobre los que se da la expansión de FinEs II.

\section{Conocimientos formados en el programa}

Resta tratar la cuestión de los conocimientos a los que acceden los alumnos del plan FinEs II, algo que por lo común no ha sido abordado por otras investigaciones. Tomando en consideración las características de la normativa y del diseño curricular, se pueden señalar en principio que esta política educativa apunta a la formación de atributos generales en un sector de la población, a partir de su acceso al nivel secundario:

- Desde la normativa que da origen al plan, empezando por la LEN hasta las resoluciones de la DGCyE en la provincia de Buenos Aires, está muy presente la importancia de una construcción de conocimientos que permitan la vida en sociedad a partir del fortalecimiento de la lectura, la comunicación, el trabajo grupal y la formación ciudadana.

- En el plan de estudios mayormente implementado en la provincia de Buenos Aires (siendo el título "Bachiller en Ciencias Sociales con orientación en políticas públicas"), se ve la importancia de las materias del área de Ciencias Sociales, Lengua y Literatura y Matemática. De la mayor carga horaria en estas asignaturas se desprende que hay un peso superior de cuestiones como la lectocomprensión, el pensamiento lógico y la ciudadanía. A su vez, los cambios normativos en el 2017 acompañan esta tendencia, ya que se da un crecimiento enfocado en Lengua y Literatura junto con Matemática (del 100\%). El resto de las materias, incluyendo las de Ciencias Sociales, crecen poco o no crecen en absoluto.

- Como contrapartida, el esquema curricular tiene una asignación horaria menor para las materias de Ciencias Naturales, Inglés e Informática. En el caso de esta última asignatura se ve una contradicción entre los enunciados legales (donde se pondera la formación de capacidades vinculadas con los avances tecnológicos recientes) y el despliegue material del programa (donde no hay insumos siquiera para poder tener una aproximación real a la informática). En simultáneo, las transformaciones del año 2017, si bien aumentan la carga de algunas materias del área de Ciencias Naturales, dejan intactas tanto a Informática como Inglés.

A su vez, se debe tener presente que en FinEs II esta formación de conocimientos generales se manifiesta a partir de una escolarización que se desarrolla de una forma diferenciada en comparación con el resto del sistema educativo y con otras ofertas escolares para jóvenes y adultos. Esto se distingue en los siguientes elementos:

- Una menor carga horaria absoluta asignada a la población destinataria. En comparación con los CENS, esta reducción pasa del 50\% en el diseño del año 2010 (Resolución 3520/10 de la DGCyE) al 31\% con la reforma del año 2017 (Resolución 713/17 de la DGCyE). Si bien esta modificación normativa agrega más tiempo de estudio, se sigue manteniendo una marcada asimetría con el resto del nivel medio.

- Peores condiciones materiales de cursada, lo cual impacta en las características de la educación garantizada. En particular, la infraestructura inadecuada y la falta de insumos hacen que el contenido obtenido sea de menor calidad. Si bien es cierto que muchas de estas cuestiones (falta de materiales, condiciones edilicias precarias, etc.) también se dan en las escuelas secundarias comunes y de la EDJA, en el caso de FinEs II el Estado prácticamente se desliga de las mismas.

- La situación laboral además es peor que la del resto de la educación secundaria común y de la EDJA, tanto para profesores como para los referentes, tomando en cuenta desde la precarización de los primeros, hasta el trabajo gratuito de los segundos. Estos factores atentan contra la calidad del programa.

Se puede ver en principio que el plan, al asegurar el derecho a la educación secundaria de ciertos sectores de la población, apunta a la formación de conocimientos generales tales como la lectocomprensión, la capacidad de razonamiento lógico, el trabajo colectivo, la autodisciplina y la conciencia 
ciudadana. Atributos que se adquieren sin embargo de modo degradado si se cotejan con el resto de las ofertas del nivel medio, contribuyendo a la diferenciación interna del sistema escolar argentino. El Estado ahorra una enorme cantidad de dinero utilizando espacios no escolares tercerizados como sedes (y no creando nuevas escuelas), reduciendo horas de la caja curricular, teniendo a los referentes de FinEs II trabajando de manera gratuita y contando con docentes abaratados por su contratación flexible. La educación de la porción de la fuerza de trabajo que se forma en el plan analizado se realiza por lo tanto de manera abaratada y diferenciada.

\section{A modo de cierre}

En este trabajo se buscó avanzar en la comprensión de la especificidad de la oferta educativa de FinEs II, en el marco de la expansión diferenciada del nivel secundario en Argentina. Para ello se examinó tanto la normativa que da forma a esta política, así como también el modo concreto en el que se realiza el proceso educativo de los jóvenes y adultos que acceden al plan en la provincia de Buenos Aires. Esto llevó a ver que el programa permite la inclusión de una porción de la fuerza de trabajo, aunque de modo degradado, en tanto las condiciones educativas son sustancialmente peores que en otros espacios de la EDJA (como es el caso de los CENS). Es entonces que se analizó como, a la vez que permite a miles de jóvenes y adultos acceder a una educación de tipo general que apunta a formar ciertos conocimientos universales, lo hace de manera diferenciada. Lejos de considerar a FinEs II como una excepcionalidad, pudo advertirse que refleja una tendencia más general de la escuela media en el territorio nacional.

Queda como un punto pendiente poder indagar sobre las demandas concretas que llevaron a la extensión del derecho a la educación media con estas características en la población destinataria. Ello requiere profundizar, entre otros aspectos, en las determinaciones específicas que hacen al sujeto que accede a FinEs II y a la EDJA, así como también en las determinaciones materiales más generales que llevaron a la expansión y diferenciación de la secundaria en Argentina.

\section{Referencias bibliográficas}

Acosta, F. (2012). La escuela secundaria argentina en perspectiva histórica y comparada: modelos institucionales y desgranamiento durante el siglo XX. Cadernos de Historia da Educação, 11(1),131-134.

Botinelli, L. (2017). Educación y desigualdad. Un repaso por algunos aportes de la sociología de la educación en la $\begin{array}{llll}\text { Argentina. } & \text { Revista } & \text { Sociedad, }\end{array}$ https://publicaciones.sociales.uba.ar/index.php/revistasociedad/article/view/2974/2463

Braslavsky, C. (1989). La discriminación educativa. Buenos Aires: Miño y Dávila Editores.

Brusilovsky, S. y Cabrera, M.E. (2006). La normativa para educación de adultos. Una de las claves para entender la vida escolar. En S. Brusilovsky (Ed.), Educación escolar de adultos. Una identidad en construcción. Buenos Aires: NOVEDUC.

Brusilovsky, S. y Cabrera, M.E. (2012). Pedagogías de la educación escolar de adultos: una realidad heterogénea. México: CREFAL.

Burgos, A. (2015). Jóvenes, educación y trabajo. Acerca del Plan FinEs como una estrategia de inclusión social y laboral [Ponencia]. 120 Congreso Nacional de Estudios del Trabajo. Buenos Aires, Argentina. https://www.aset.org.ar/2015/ponencias/7 Burgos.pdf

De la Fare, M. (2013). Estudiantes del nivel secundario de la Educación Permanente de Jóvenes y Adultos (EPJA). Serie Informes de Investigación № 8. Argentina: ME-DiNIECE.

De Luca, R. (2017). Brutos y baratos. Descentralización y privatización en la educación argentina (1955-2001). Ciudad Autónoma de Buenos Aires: Ediciones RyR.

Esper, T. (2017). Educación Secundaria: obligatoriedad, expansión y segmentación. Una evaluación del plan FinEs 2 como alternativa al formato tradicional. RELAPAE, (7), 31-42. https://revistas.untref.edu.ar/index.php/relapae/article/view/85/72

Facioni, C.; Ostrower, L. y Rubisztain, P. (2013). Programas de terminalidad educativa en Argentina: aportes para un análisis crítico del "Plan de Finalización de Estudios Primarios y Secundarios" (Plan FinEs) [Ponencia]. X Jornadas de Sociología de la UBA, Buenos Aires, Argentina. https://cdsa.aacademica.org/000-038

Finnegan, F. (2016). La educación secundaria de jóvenes y adultos en la Argentina. Una expansión controversial.

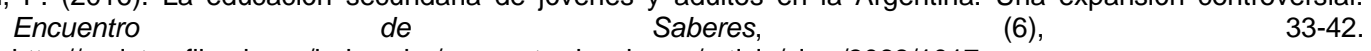
http://revistas.filo.uba.ar/index.php/encuentrodesaberes/article/view/3068/1017

Finnegan, F. y Brunetto, C. (2014). "Acá hay un trabajo político": Actores y relaciones en la producción del Plan FinEs 2 Secundaria en la Provincia de Buenos Aires [Ponencia]. VIII Jornadas de Sociología de la UNLP, Buenos Aires, Argentina. http://www.memoria.fahce.unlp.edu.ar/trab_eventos/ev.4364/ev.4364.pdf

Finnegan, F. y Montesinos, P. (2016). Panorama de la oferta educativa del Nivel Secundario de la modalidad Educación Permanente de Jóvenes y Adultos en la Provincia de Buenos Aires. Serie Apuntes de Investigación N. 3. Ciudad Autónoma de Buenos Aires: MED-DiNIECE.

Giménez, D. e Inda, M. (2015). Condiciones y regulaciones del puesto de trabajo docente al interior del Plan FinEs. En V. Seoane (Coord.). III Seminario Nacional de la Red Estrado. Formación y trabajo docente: aportes a la democratización educativa, 216-228. Facultad de Humanidades y Ciencias de la Educación, UNLP. https://libros.fahce.unlp.edu.ar/index.php/libros/catalog/book/67

González Porcella, C. y Pereira, G. (2015). El trabajo docente en los nuevos formatos escolares en la educación de adultxs en el marco de la obligatoriedad de la escuela secundaria en Argentina: Programa FinEs 2 y Bachilleratos Populares. En V. Seoane (Coord.). III Seminario Nacional de la Red Estrado. Formación y trabajo docente: aportes a la democratización educativa, 1535-1551. Facultad de Humanidades y Ciencias de la Educación, UNLP. https://libros.fahce.unlp.edu.ar/index.php/libros/catalog/book/67

Hirsch, D. (2020). Reformas de la educación técnica y transformaciones productivas: el vínculo entre el Estado, las empresas y las escuelas para la formación de la fuerza de trabajo (1992-2014) [Tesis de doctorado no publicada]. Universidad de Buenos Aires. 
REVISTA DE LA ESCUELA DE CIENCIAS DE LA EDUCACIÓN, AÑO 17, NRO. 16, VOL. 2, JULIO A DICIEMBRE DE 2021. PÁGINAS 26-36. ISSN 2362-3349 (EN LÍNEA). INCLUSIÓN Y DIFERENCIACIÓN DE LA EDUCACIÓN SECUNDARIA PARA JÓVENES Y ADULTOS EN ARGENTINA. UN ANÁLISIS SOBRE LA ESPECIFICIDAD DE LA OFERTA DEL PLAN FINES II. JULIÁN OLIVARES.

Hirsch, M. e Iñigo, L. (10-12 de agosto de 2005). La formación del sistema educativo argentino: ¿producción de fuerza de trabajo vs. producción de ciudadanos? [Ponencia]. $7^{\circ}$ Congreso Nacional de Estudios del Trabajo, Buenos Aires, Argentina. https://www.aset.org.ar/congresos/7congreso.htm

Huenchunao, V. y Muñoz, H. (2015). La configuración del trabajo docente pedagógico desde las nuevas relaciones laborales en la escuela media de la norpatagonia: el caso Plan FinEs. En V. Seoane (Coord.). III Seminario Nacional de la Red Estrado. Formación y trabajo docente: aportes a la democratización educativa, 98-110. Facultad de Humanidades y Ciencias de la Educación, UNLP. https://libros.fahce.unlp.edu.ar/index.php/libros/catalog/book/67

Iñigo, L. y Río, V. (2017). Extensión de la escolaridad y obligatoriedad de la escuela secundaria en Argentina: el papel de la universalización de la lectura y escritura. Universitas humanística, 83, 206-236. http://dx.doi.org/10.11144/ Javeriana.uh83.eeoe

Komañski, F. (2016). Precariedad y acción colectiva: Obstáculos y limitaciones para la conformación de espacios de organización docente en el Plan FinEs II La Plata [Tesis de grado, Universidad Nacional de La Plata]. Repositorio institucional de la Facultad de Humanidades y Ciencias de la Educación de la Universidad Nacional de La Plata. http://www.memoria.fahce.unlp.edu.ar/tesis/te.1296/te.1296.pdf

Kurlat, S. y Lozano, P. (3-5 de diciembre de 2014). Plan FINES 2S: Contribuciones y limitaciones al logro de la inclusión en el nivel secundario de jóvenes y adultos [Ponencia]. VIII Jornadas de Sociología de la UNLP, Buenos Aires, Argentina. http://www.memoria.fahce.unlp.edu.ar/trab_eventos/ev.4557/ev.4557.pdf

Levy, E. y Rodríguez, L. (2015). La educación, las políticas públicas y los procesos de constitución de sujetos en la última década. Espacios de Crítica y Producción, 51, 3-12. http://revistascientificas.filo.uba.ar/index.php/espacios/article/view/1866

López, E. (24-26 de junio de 2015). El formato escolar en políticas de inclusión educativa: el caso del FINES 2 [Ponencia]. $\mathrm{V}$ congreso Nacional e Internacional de estudios comparados en Educación, Buenos Aires, Argentina. http://www.saece.com.ar/docs/congreso5/trab090.pdf

Mendonça, M. y Pérez Trento, N. (2020). El devenir del sistema universitario público en la Argentina a través de sus olas expansivas: Diferenciación en la formación de fuerza de trabajo y acumulación de capital. (1971-2015). Archivos de Políticas Educativas, 28(49). https://doi.org/10.14507/epaa.28.4914

Montesinos, M.; Schoo, S. y Sinisi, L. (2010). Aportes para pensar la Educación de Jóvenes y Adultos en el nivel secundario. Un estudio desde la perspectiva de los sujetos. Serie La Educación en Debate ํำ 7. ME-DiNIECE.

Olivares, J. (2018). La inclusión como forma de la diferenciación. Educación y trabajo en el plan de terminalidad secundaria FINES II en Moreno (2010-2017) [Trabajo final de grado, Universidad Nacional de Luján]. Repositorio Digital Institucional de Acceso Abierto de la Universidad Nacional de Luján. https://ri.unlu.edu.ar/xmlui/handle/rediunlu/429

Río, V. y Schoo, S. (2016). La educación secundaria obligatoria en los procesos de reforma contemporáneos. Políticas nacionales y acuerdos federales en torno a su universalización. En Ruiz, G. (Comp.). La educación secundaria en el marco de las reformas educativas nacionales. Regulaciones federales y políticas jurisdiccionales, 131189. Buenos Aires: Eudeba.

Riquelme, G.; Herger, N. y Sassera, J. (2018). Deuda social educativa con jóvenes y adultos. Entre el derecho a la educación, los discursos de las políticas y las contradicciones de la inclusión y la exclusión. Buenos Aires: Editorial de la Facultad de Filosofía y Letras, UBA.

Rivas, A. y Dborkin, D. (2018). ¿Qué cambió en el financiamiento educativo en Argentina? Documento de trabajo N.․ 162. Ciudad Autónoma de Buenos Aires: CIPPEC.

Rodríguez, L. (2009). Educación de adultos en la historia reciente de América Latina y el Caribe. Revista Electrónica de $\begin{array}{llllll}\text { Educación } y & \text { Formación Continua de }\end{array}$ http://campus.usal.es/ efora/efora_03/monografico_efora_03.pdf

Tiramonti, G. (2009). Una aproximación a la dinámica de la fragmentación del sistema educativo argentino. Especificaciones teóricas y empíricas. En Montes, N. y Tiramonti, G. (Comps.). La escuela media en debate. Problemas actuales y perspectivas desde la investigación, 25-38. Manantial/FLACSO.

Tiramonti, G. (2019). La escuela media argentina: el devenir de una crisis. Propuesta Educativa, 51, 78-92. http://propuestaeducativa.flacso.org.ar/wp-content/uploads/2019/11/PropuestaEducativa51-articuloTIRAMONTI.pdf

Viego, V. (2015). Políticas públicas para la terminalidad educativa: el caso de Plan Fines en Argentina. Archivos Analíticos de Políticas Educativas, 23(116). http://dx.doi.org/10.14507/epaa.v23.1929

\section{Marco normativo y fuentes}

Convenio Marco 299/08 del Ministerio de Educación de la Nación.

Disposición 27/11 de la Dirección General de Cultura y Educación de la provincia de Buenos Aires.

Informe № 96 de la Jefatura de Gabinete de Ministro al Senado (2016).

Ley Nacional № 26.206.

Ley Provincial № 13.688 .

Resolución 118/10 del Consejo Federal de Educación.

Resolución 146/2020 del Ministerio de Educación de la Nación.

Resolución 178/16 del Ministerio de Educación de la Nación.

Resolución 22/07 del Consejo Federal de Educación.

Resolución 2280/17 de la Dirección General de Cultura y Educación de la provincia de Buenos Aires. Resolución 3520/10 de la Dirección General de Cultura y Educación de la provincia de Buenos Aires.

Resolución 4122/08 de la Dirección General de Cultura y Educación de la provincia de Buenos Aires. Resolución 444/12 de la Dirección General de Cultura y Educación de la provincia de Buenos Aires. Resolución 6321/95 de la Dirección General de Cultura y Educación de la provincia de Buenos Aires. Resolución 66/08 del Consejo Federal de Educación.

Resolución 713/17 de la Dirección General de Cultura y Educación de la provincia de Buenos Aires.

Resolución 87/09 del Consejo Federal de Educación.

Resolución 917/08 del Ministerio de Educación de la Nación. 